\title{
Concreteness and encoding instructions in paired-associate transfer
}

\author{
JOHN H. MUELLER and JAMES P. PICKERING \\ University of Missouri, Columbia, Missouri 65201
}

\begin{abstract}
Subjects were instructed to use either different mnemonics (compound images or verbal combinations) on List 1 and List 2 in an A-B, A-Br transfer paradigm, or to use the same type of mnemonic strategy for both tasks. Compared to A-B, C-B control subjects who were similarly instructed, there was no evidence that switching the type of elaborative encoding led to reduced interference.
\end{abstract}

Negative transfer is typically observed when subjects are required to learn new responses to old nominal stimuli, as in the A-B, A-Br transfer paradigm. Such transfer is usually attributed to interference from List 1 associations. It has been argued, however, that subjects might select new functional stimuli during List 2, thus avoiding interference (Bryk \& Kausler, 1966). Martin (1968) has developed an encoding variability hypothesis which argues that recoding will be easier when the stimulus has multiple encodings.

Two things are involved in this hypothesis: (1) the assumption of multiple encoding per se and (2) the assumption that subjects actually recode to avoid interference; the latter will be referred to as the recoding corollary. It seems reasonable that encodings can vary for a given nominal stimulus, but the support for the recoding corollary has been mixed when stimulus attributes alone are varied (Mueller, Brown, \& Kausler, 1975). The evidence, while rather limited, seems more positive when the recoding manipulation involves the whole pair, stimulus and response. For example, the free pairing procedure utilized by Brown and Read (1970) more consistently yields reduced interference (e.g., Read, 1974). Ellis (1973) has found response representativeness to be an important factor in a series of studies, and it may be that free pairing allows this factor to work optimally, whereas manipulation of stimulus properties alone may not as effectively induce recoding.

The present research investigated (1) a property of stimulus terms which could affect recoding and (2) the role of mnemonic instructions in paired-associate transfer. First, while Martin (1968) identified encoding variability as inversely related to meaningfulness, it also seems that the concreteness of the stimulus term might determine encoding options. Paivio (1971) has argued that concrete (high-imagery) items may be processed

Requests for reprints should be sent to J. H. Mueller, Psychology Department, 210 McAlester Hall, University of Missouri, Columbia, Missouri 65201. The authors wish to acknowledge the contributions of Donald Kausler and Sam Brown at various stages in this research, plus the assistance of Larry Beigel, Stuart Brown, and Margaret Lineberry as experimenters. through either an imaginal or a verbal-symbolic processing system, or both, while abstract (low-imagery) items are processed through the verbal-symbolic system alone. This "dual-code" analysis can be readily related to the recoding corollary. For example, an image could be utilized in the first task and then the same nominal stimulus could be encoded verbally in the second task, or a different image might be used in the second task, with interference potentially reduced in either manner. Abstract items presumably would not have sucn flexibility.

The effect of elaborative encodings on recoding can also be examined in terms of imaginal processes. The "conceptual pegs" hypothesis (Paivio, 1971) assumes that subjects form a compound image incorporating both the stimulus and response term of a pair, so that the later presentation of the stimulus alone readily generates the response. In terms of the recoding corollary, subjects could be encouraged via mnemonic instructions to generate a compound image for List 1 and then a sentence or phrase for List 2. Even without special instructions, though, the conceptual pegs notion seems compatible with the analysis of the role of response features in cue selections (cf. Davis, Brown, \& Ritchie, 1968; Ellis, 1973), since compound images would involve both the stimulus and response terms.

In spite of the voluminous literature on imagery and mnemonics, we found very little bearing upon their effect in two-stage paired-associate transfer. Bower and Reitman (1972) found that progressive elaboration of images reduced retroactive inhibition with peg-word and loci mnemonics in multilist free recall. To the extent that such procedures correspond to the A-B, A-C pairedassociate transfer paradigm, then, those results seem encouraging. Research in our laboratory (Pickering, Note 1) found that high-imagery stimuli generally led to fewer errors during List 2 learning for the $\mathrm{A}-\mathrm{Br}$ paradigm, as would be expected if subjects were able to recode functional stimuli to avoid interference. However, the magnitude of the effect was rather small compared to the usual effect of manipulations in imagery level, and its interpretation was complicated by the fact that stimulus imagery was also effective during 
List 2 for the control paradigm. Instructions prior to List 2 suggesting the formation of images or verbal combinations also increased transfer, but did so equally for the interference and control paradigms, and independent of imagery level. The experiments to be reported here were conducted to examine the effect of instructional inducements to switch or maintain a given type of mnemonic strategy for List 1 and List 2 of an interference transfer paradigm, and to provide further data on the effect of stimulus imagery in transfer.

\section{METHOD}

\section{Experiment 1}

Design and subjects. The design was a 2 by 3 factorial, with Paradigms ( $\mathrm{A}-\mathrm{Br}, \mathrm{C}-\mathrm{B}$ ) and Instructions (change mnemonics between Lists 1 and 2, use the same mnemonic for Lists 1 and 2, no instructions) as between-subjects factors, with 12 subjects from introductory psychology classes in each group.

Materials. The lists were constructed using 60 concrete nouns as stimuli and responses. All items had a Thorndike-Lorge frequency of 35 or greater and rated imagery greater than 5.76 (Paivio, Yuille, \& Madigan, 1968). Three basic 10-pair lists were constructed (A-B, C-B, A-Br), with two versions of each. These were arranged in four different random orders for study and four additional orders for tests.

Procedure. Each subject learned two different 10-pair lists, with either the same responses but different stimuli (A-B, C-B) or the same stimuli and the same responses re-paired (A-B, A-Br) for Lists 1 and 2. Acquisition was by the recall method, using a 4-sec rate of presentation. Learning was to a criterion of one errorless trial for each task, with an inter-task interval of about $3 \mathrm{~min}$. Paradigmatic variation was accomplished by using different first lists, with the test list (A-B) the same for all subjects.

One-third of the subjects with the A-Br paradigm were told to form a compound image of the stimulus and response for each pair during List 1 , and then to form verbal combinations during List 2 , or vice versa. An example of each strategy was given as appropriate, though subjects were not told about the alternative strategy until just prior to List 2. It was expected that these changed subjects could recode and show reduced negative transfer. Since the change in type of mnemonic might itself have a detrimental effect, offsetting any recoding advantage, a third of the C-B subjects were also told to change for purposes of comparison. Another third of the subjects with each paradigm were told to use the same type of mnemonic strategy for both lists, half being instructed to form images in each list and half instructed to form verbal combinations. These subjects should not show reduced negative transfer and could show increased negative transfer, according to encoding variability theory. The remaining subjects with each paradigm were uninstructed, i.e., they received standard paired-associate learning instructions, with no mention of either imaginal or verbal mnemonics.

Following the second task, subjects were asked to rate the effectiveness of any special instructions and their general usage of imagery. The $\mathrm{A}-\mathrm{Br}$ subjects were then shown the stimuli one at a time and asked to state for each whether they changed encodings for Lists 1 and 2. Those stimuli for which subjects claimed to switch should show the greatest reduction in negative transfer (Polzella \& Martin, 1973).

\section{Experiment 2}

The procedure was identical to Experiment 1, except that the pairs were composed of abstract nouns as stimuli and responses (Thorndike-Lorge frequencies greater than 35 and rated imagery less than 3.76, Paivio, et al, 1968).

\section{RESULTS $^{1}$}

\section{Experiment 1}

The results of Experiment 1 are summarized in Table 1. There was no Paradigm main effect or Paradigm by Instructions interaction for List 1 errors (Fs $<1.71)$, but the main effect of Instructions was significant $[\mathrm{F}(2,66)=3.31, \mathrm{MSe}=81.59]$, as uninstructed subjects made more errors than those with some mnemonic instructions. There was no difference on List 1 for subjects whose strategy would be changed on List 2 vs. those who would maintain the same strategy.

The Instructions main effect was significant for List 2 errors $[F(2,66)=3.28, \quad \mathrm{MSe}=20.77]$. Uninstructed subjects made more errors than the changed and unchanged subjects (ps $<.05$ ), and the latter two conditions did not differ. There was no Paradigm by Instructions interaction $(F<1)$, though there was the expected performance deficit for the $\mathrm{A}-\mathrm{Br}$ paradigm $[F(1,66)=5.91, \mathrm{MSe}=20.77]$. The results were the same for errors early in List 2 , as the Instructions and Paradigms main effects were significant $[F(2,66)=9.72$ and $F(1,66)=4.13$, respectively, $\mathrm{MSe}=9.84]$, with no interaction $(F<1)$.

For the $\mathrm{A}-\mathrm{Br}$ subjects, total errors were reclassified into errors involving stimuli that subjects identified as those for which they changed encodings vs. those encoded the same way on Lists 1 and 2 . The average number of changed stimuli was $7.75,7.75$, and 8.17 , and the average number of List 2 errors per stimulus was .41 , .49 , and .62 , for the changed, unchanged, and uninstructed $\mathrm{A}-\mathrm{Br}$ groups, respectively. The Instructions main effect was not significant in either case $(F s<1)$. The average number of List 2 errors per unchanged stimulus for these three groups was $.38, .58$, and $.70(\mathrm{~F}<1)$.

The following transfer score was computed for each subject: (List 1 errors - List 2 errors)/(List 1 errors + List 2 errors) $\times 100$. The $\mathrm{A} \cdot \mathrm{Br}$ paradigm was significantly worse than the C-B paradigm $[\mathrm{F}(1,66)=12.28$,

Table 1

Summary Statistics for Experiment 1 by Paradigm and Instructions

\begin{tabular}{lrrrrrrr}
\hline & \multicolumn{3}{c}{ A-Br Paradigm } & & \multicolumn{3}{c}{ C-B Paradigm } \\
\cline { 2 - 3 } \cline { 6 - 8 } & Changed & Same & None & Changed & Same & None \\
\hline List 1 Errors & 2.83 & 5.75 & 6.08 & & 3.67 & 3.25 & 13.08 \\
List 2 Frrors & 4.17 & 5.58 & 6.83 & & 1.67 & 1.67 & 5.42 \\
List 2 Errors* & 4.00 & 4.50 & 5.83 & & 1.58 & 1.42 & 4.42 \\
Transfer Score & -33.85 & -1.27 & -4.63 & & 37.38 & 41.25 & 24.04 \\
\hline
\end{tabular}

*Trials 1 and 2 
Table 2

Summary Statistics for Experiment 2 by Paradigm and Instructions

\begin{tabular}{|c|c|c|c|c|c|c|}
\hline & \multicolumn{3}{|c|}{ A-Br Paradigm } & \multicolumn{3}{|c|}{ C-B Paradigm } \\
\hline & Changed & Same & None & Changed & Same & None \\
\hline $\begin{array}{l}\text { List } 1 \text { Errors } \\
\text { List } 2 \text { Errors } \\
\text { List } 2 \text { Errors* } \\
\text { Transfer Score }\end{array}$ & $\begin{array}{r}37.08 \\
53.50 \\
14.42 \\
-18.72\end{array}$ & $\begin{array}{r}27.67 \\
38.58 \\
14.50 \\
-19.43\end{array}$ & $\begin{array}{r}27.58 \\
39.75 \\
15.25 \\
-19.26\end{array}$ & $\begin{array}{l}39.25 \\
18.92 \\
11.00 \\
23.67\end{array}$ & $\begin{array}{r}27.42 \\
16.17 \\
9.58 \\
27.14\end{array}$ & $\begin{array}{l}47.33 \\
25.33 \\
13.42 \\
27.93\end{array}$ \\
\hline
\end{tabular}

*Trials 1 and 2

$\mathrm{MSe}=3,304.29$ ], but there was no Instructions main effect or Paradigm by Instructions interaction $(F s<1)$.

\section{Experiment 2}

The results of Experiment 2 are shown in Table 2. No effects were significant for List 1 errors (Fs $<1.31$, MSe $=714.00$ ).

The A-Br paradigm led to more List 2 errors than the C-B paradigm $[\mathrm{F}(1,66)=16.60, \mathrm{MSe}=614.48]$, but there was no Instructions main effect or Paradigm by Instructions interaction ( $\mathrm{Fs}<1.01)$. The $\mathrm{A}-\mathrm{Br}$ paradigm also led to more errors on the first two trials of List 2 $[\mathrm{F}(1,66)=14.12, \mathrm{MSe}=14.64]$, with no Instructions main effect $[F(2,66)=2.28, p<.12]$, or Paradigm by Instructions interaction $(\mathrm{F}<1)$.

The transfer scores revealed the expected deficit for the A-Br paradigm $[\mathrm{F}(1,66)=37.80, \mathrm{MSe}=979.44]$, with no Instructions main effect or Paradigm by Instructions interaction $(\mathrm{Fs}<1)$.

Dividing total errors in the $\mathrm{A}-\mathrm{Br}$ groups into those involving changed or unchanged encodings revealed that the average number of changed stimuli was 5.92, 4.58, and 6.28 , for the changed, unchanged, and uninstructed A-Br groups, respectively; and the average number of List 2 errors per changed stimulus was 5.49, 3.30, and 3.73 , for the changed mnemonics, unchanged, and uninstructed groups, respectively. For the same three groups, the average number of List 2 errors for unchanged stimuli was $2.76,2.49$, and 3.43 . The Instructions effect was not significant in either case $(\mathrm{Fs}<1.04)$.

\section{DISCUSSION}

The recoding analysis suggested that subjects would show reduced negative transfer on List 2 when they were encouraged to use different mnemonics on Lists 1 and 2 . However, switching between verbal and imaginal mnemonics did not significantly reduce interference, as indicated by the absence of Paradigm by Instructions interactions in Experiments 1 and 2.

While the present results may at first seem at variance with the findings of Bower and Reitman (1972), there is one procedural difference that may be responsible. Specifically, the present manipulations did not involve progressive incorporation of List 1 and 2 responses in the image or phrase, since our interest was in the effect of using different encoding strategies for each list. The latter seems to more directly test the recoding corollary, though the former may functionally approximate different encodings. Our methods may correspond better to Bower and Reitman's separate-images group, and our conclusions are based upon comparisons to uninstructed groups. Since they did not include an uninstructed condition, it is not possible to compare the two studies further. Progressive elaboration may reduce interference, but different elaborations did not seem to do so.

As regards imagery as a stimulus attribute, the uninstructed $\mathrm{A}-\mathrm{Br}$ and $\mathrm{C}-\mathrm{B}$ groups of each experiment are most pertinent. There was some evidence that concrete items led to less negative transfer-for the $\mathrm{A}-\mathrm{Br}$ paradigm, but the interpretation is complicated by differential degrees of List 1 learning, and the effect is rather small. In an article which appeared after the present experiments were completed, Parker and Bass (1975) reported no effects in transfer due to stimulus imagery when words and pictures were compared. Furthermore, some authors have argued that abstract items have more variable encodings than concrete items (Galbraith, 1975; Paivio, 1971, p. 287), just the opposite of the assumption outlined above. While the dual-coding analysis seems to us to suggest that concrete items might be more flexibly encoded, other conceptualizations are clearly possible, and the data to this point are equivocal.

\section{REFERENCE NOTE}

1. Pickering, J. P. A test of concreteness as a variable in Martin's encoding variability hypothesis. Unpublished Master's thesis, University of Missouri, 1972.

\section{REFERENCES}

Bower, G. H., \& Reitman, J. S. Mnemonic elaboration in multilist learning. Journal of Verbal Learning and Verbal Behavior, 1972, 11, 478-485.

Brown, S. C., \& READ, J. D. Associative organization in paired-associate learning and transfer. Journal of Verbal Learning and Verbal Behavior, 1970, 9, 317-326.

BryK, J. A., \& Kausler, D. H. Stimulus meaningfulness and unlearning in the A-B, A-C transfer paradigm. Journal of Experimental Psychology, 1966, 71, 917-920.

Davis, W. L., Brown, S. C., \& Ritchie, E. Cue selection as a function of degree of learning and response similarity. Journal of Experimental Psychology, 1968, 78, 323-328.

Ellis, H. C. Stimulus encoding processes in human learning and memory. In G. H. Bower (Ed.), Psychology of learning and motivation (Vol. 7). New York: Academic Press, 1973.

Galbraith, R. C. Encoding variability and the concreteness effect in paired-associate learning. Memory \& Cognition, 1975, 3, 282-286.

Martin, E. Stimulus meaningfulness and paired-associate transfer: An encoding variability hypothesis. Psychological Review, 1968, 75, 421-441.

Mueller, J. H., Brown, S. C., \& Kausler, D. H. Pairedassociate transfer with homograph stimuli. Journal of Experimental Psychology: Human Learning and Memory, 1975, 104, 167-172.

PaIvio, A. Imagery and verbal processes. New York: Holt, Rinehart, \& Winston, 1971. 
Paivio, A., Yuille, J. C., \& Madigan, S. Concreteness, imagery, and meaningfulness values for 925 nouns. Journal of Experimental Psychology, 1968, 76(1, Pt. 2).

PARKer. J. F.. \& Bass, D. Pictures versus words as stimuli in paired-associate transfer. American Journal of Psychology, 1975, 88, 635-642.

Polzella. D. J., \& Martin, E. Stimulus encoding in A-B, A-D transfer. American Journal of Psychology, 1973, 86, 589-600.

READ. J. D. Free-pairing, imagery, and proactive inhibition in multilist paired-associate learning. Psychological Reports, 1974, 34. 267-272.

\section{NOTE}

1. Unless otherwise noted, effects described as significant have $\mathrm{p}<.05$ or greater.

(Received for publication June 21, 1976.) 